Lynah for diagnostic and therapeutic purposes. Chaulmoogra oil and the mixed esters of chaulmoogra oil injected intratracheally into rabbits produces distinct pathologic changes in the lungs which may range from a profound acute pneumonic consolidation with abscess formation to a proliferative bronchopneumonia, depending on the concentration and the localization of these oils in the lungs. A concentration of these oils as low as 10 per cent. in olive or liquid petrolatum, injected intratracheally, still produces a distinct proliferative bronchopneumonia. Olive oil and liquid petrolatum injected intratracheally into rabbits in small amounts (not to exceed 1 c.c.) are readily aspirated into the finest pulmonary divisions-the alveoli-to be retained for a relatively long time (months), where they can produce a mild type of a proliferative bronchopneumonia. This study verifies and emphasizes previous observations by others indicating that great care is necessary in the introduction of any foreign substances into the lungs normally, and especially in pulmonary diseases. ${ }^{20}$ It has been suggested, but scientific proof is lacking, that the fibrotic changes subsequent to the injection of certain foreign substances into diseased foci in the lungs may have a beneficial action on the diseased area (lung abscess or bronchiectasis). It is questionable also whether even local application, as is accomplished by use of the bronchoscope, will insure that the foreign substance may not leave the focus of intended localization to exert a deleterious action on normal lung tissue some distance away. These questions require further detailed study.

\section{DEVELOPMENT AND CORRECTION OF EXTENSIVE CYSTS OF THE MAXILLAE}

ANATOMIC AND PROSTHETIC FACTORS *

HUGH W. MacMillaN, D.D.S., M.D. CINCINNATI

In Black's "Special Dental Pathology," under the heading of chronic osteitis of the maxillae, it is stated that the number of cases of chronic osteitis which have occurred about the roots of upper lateral incisors have each year attracted the attention of the oral surgery department of Northwestern University. Black then gives several reasons why the pulp of the superior lateral incisors is more liable to be destroyed than that of any of the remaining superior teeth. His observations regarding the frequency of chronic osteitis occurring about the roots of superior lateral incisors are equally true of the frequency of radicular cyst formations of infective origin in this particular region. But he does not explain why chronic osteitis or extensive cyst formations involving the antrum and nasal fossae very rarely originate from abscessed centrals, cuspids and bicuspids. Nor does he record any differences in the extent of the bone destruction or any peculiarities in the osteology which might predispose certain teeth to cause extensive destructive bone lesions.

The consideration of the anatomic environment of the superior lateral incisor region affords a further

20. This study must not be misconstrued as a personal criticism of Lukens and Lynah; but it emphasizes the necessity for experimental studies before applying diagnostic or therapeutic procedures clinically. The complete report of this study will appear elsewhere.

${ }^{*}$ Read before the Section on Stomatology at the Seventy-Third Annual Session of the American Medical Association, St. Louis,
May, 1922. explanation of the phenomenon. The path of discharge of alveolar abscesses depends as a rule on the character of the bone surrounding the apex or apexes of the tooth. For example, alveolar abscesses arising from the ten superior anterior teeth other than the superior lateral and first bicuspid invariably discharge through the external plate, as the apexes are usually covered by a very thin layer of bone, which is easily broken down. Often the bone is entirely absent from over the apexes of these teeth, and apical infection is at once heralded by a parulis which is immediately

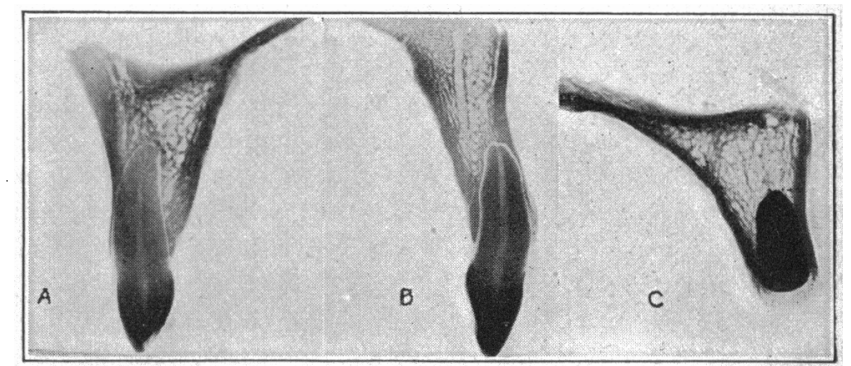

Fig. 1. $A$ and $C$ show the typical relations of the superior lateral incisor. The apex is usually completely surrounded by cancellous tissue, which, in turn, is bounded by the dense, compact internal and external alveolat plates. The heavy, dense external plate is shown in $B$ Owing to these relations, this area is predisposed to extensive destruccyst formation, on account of the area which may be affected before cyst formation, on account of the area which may be affected before a parulis or fistulas, or by bulging of the alveolar plates.

diagnosed. Unlike the apex of any other superior tooth, that of the superior lateral incisor is usually completely surrounded by cancellous tissue, and is more often situated midway between the outer and inner alveolar plates. Owing to this anatomic arrangement, an alveolar abscess arising from this tooth does not easily discharge, on account of the relatively greater distance to the surface. As this condition usually originates from a chronic periapical infection, the destructive process is very slow and of long standing. If cyst formation occurs, the surrounding part is soon under pressure from the contained fluid. As the cyst takes the line of least resistance, the cancellous tissue is easily destroyed, the alveolar plates are bulged out and the cyst slowly attains enormous proportions. Since there is rarely pain, the patient is not aware of the presence of the cyst until deformity results. The

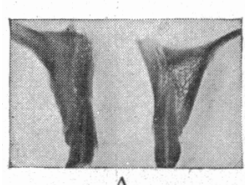

$\mathrm{A}$

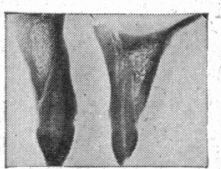

B

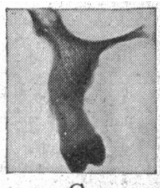

C
Fig. 2. $A$, comparison of the periapical region of a superior central incisor (showing result of periapical infection) with that of a superior lateral incisor; $B$, superior cuspid and superior lateral; $C$, superior sec ond bicuspid. The apexes of the superior central incisor, cuspid, buccal root of first bicuspid and second bicuspid are either covered by a very thin external alveolar plate or have no osseous covering. Periapical infections incident to these teeth are immediately diagnosed by the patient from the formation of a subperiosteal abscess.

cyst is then usually lanced by the attending physician. I do not recall a case in which the cyst discharged of its own accord.

The lingual root of the superior first bicuspid is situated similarly to that of the superior lateral incisor in its relation to the buccal and lingual alveolar plates. The same mechanical problem presents itself to periapical infections of this root. The same extensive 
destruction should be expected and does occur. The bone destruction is not ustually so extensive or so long standing, as the trouble is usually detected by the simultaneous infection of the buccal root, over which the buccal alveolar process is often absent. These cyst formations from the lingual root are the cause of

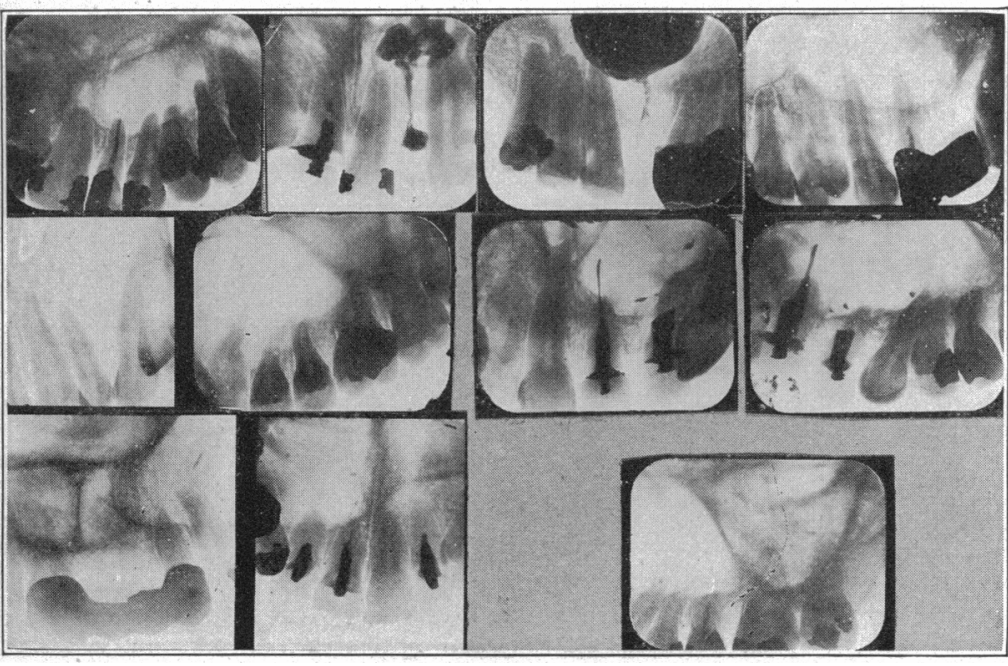

Fig. 3.-Typical extensive bone destruction in upper jaw arising from the superior be covered by the restoration, the better the retention of the denture.

The success of the future restoration will depend to a great extent on the conservative treatment of the alveolar plates. Only as much of the buccal alveolar plate should be removed as is necessary for the complete extirpation of the cyst membrane. By all means, the part of the bone giving attachment to the buccinator should be retained. The crest of the lingual plate should be trimmed back from 2 to $5 \mathrm{~mm}$., in order to prevent a wedge-shaped alveolar ridge.

Following the removal of the cyst membrane, an opening should be made into the inferior meatus, and all drainage maintained through the nose. The buccal and lingual flaps should be brought together, completely closing the cyst cavity from the mouth. If the technic regarding the preparation of the buccal flap is followed, not only may complete closure be obtained, but also a foundation for a future ridge with a buccal fornix of normal depth, which is of inestimable benefit for the future prosthesis.

\section{CONCLUSION}

Special emphasis should be placed on the many mistaken diagnoses and considerable inconvenience to the patient. They usually terminate by breaking through the lingual plate, causing a bulging in the roof of the mouth. The swelling is usually lanced several times before the cause is detected as coming from the lingual root.

Clinically, radicular cysts of the maxilla vary from those the size of a grain of wheat to those which occupy the whole cancellous tissue of the maxilla, bulging out the external and internal plates, obliterating the roof of the mouth, invading the nasal fossae and completely occupying the maxillary antrum.

The surgical correction of these enormous cysts should be accomplished with the mechanical requirements of the future prosthesis in mind. With some slight modifications, the classic operation of removal of the cyst intra-orally and draining through the inferior meatus is practiced exclusively in the treatment of cysts involving the antrum. The buccal incision is made just above the gum margin, and the mucoperiosteal tissue is carefully raised from the bone up to the attachment of the buccinator muscle. With curved scissors, the mucous and submucous tissues are dissected from the buccal fornix as far as is necessary to secure sufficient tissue for complete closure. It is important that the buccinator muscle be not included in the

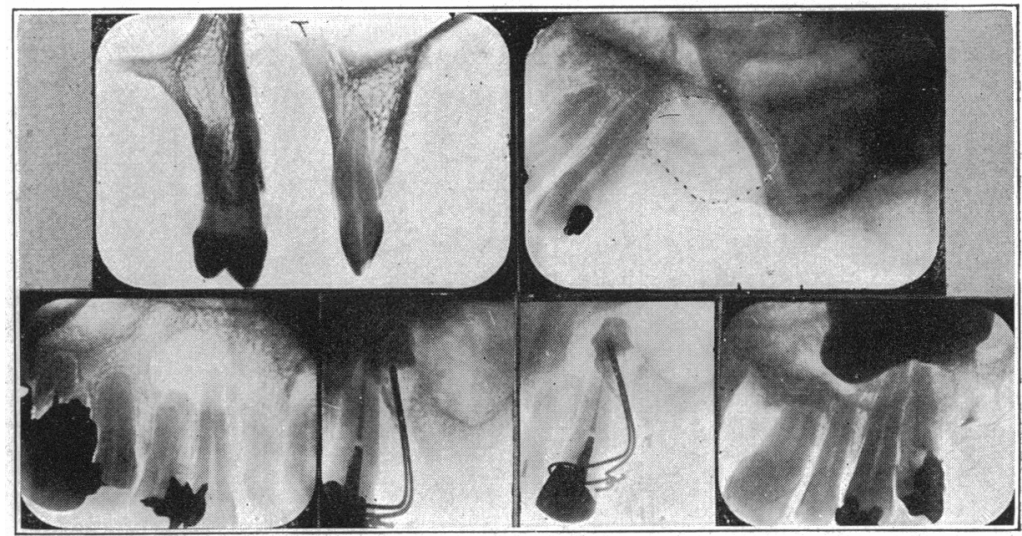

Fig. 4.-Bony relations of the lingual root of the superior first bicuspid are shown above, at left. Remaining films show typical bone destruction resulting from infections of the lingual root. importance of a knowledge of the anatomy of the periapical regions in the successful treatment of diseases arising from dental infections. The peculiar environment of the superior lateral incisor and superior first bicuspid makes the prognosis of alveolar infections decidedly different from that of the remaining anterior superior teeth. In the correction of extensive cysts arising from the superior lateral incisor, a knowledge of the principles of prosthesis, combined with those of plastic surgery, enables the operator to restore the part to its original appearance and function. flap, as bringing it down over the future alveolar crest makes it impossible to secure a proper foundation for a prosthetic restoration, as the denture will be dislodged whenever the muscle contracts. By dissecting the muscle from the flap, it will be found that, eventually, the buccal fornix on the affected side will be the same depth as before. This is of great importance, as the more extensive the area to
Dr. Arthur D. Black, Chicago: My clinical observation does not agree with the statements made by Dr. MacMillan relative to the penetration of pus in the formation of sinuses in the regions he mentioned. It will be worth while for Dr. MacMillan and others to make the most critical study of the bony structures in relation to the position of the roots of the teeth in a larger number of cases than I imagine he 
has done up to the present time, in order that we may have more definite ideas than most of us have today. Dr. MacMillan made reference to statements in my father's book on the relationship of the upper lateral incisors to the infective involvements of the bone about the apex of the root. The effort was there made to explain why the pulp of the lateral incisor so frequently died, and to recite the difficulties of making a perfect root filling; both contributing to the frequency of abscess formation about the root apex. It has been my observation that a large number of cases in which the bone is involved about the apex of the root of the upper lateral have produced sinuses discharging through the gum, and I have seen more sinuses discharging through the gum to the buccal side of an upper first bicuspid than I have through the lingual side. My observation docs not agree fully with Dr. MacMillan's statement regarding the route of discharge into the antrum or into the nasal fossae. I have seen several cases in which a sinus from an upper central incisor discharged into the nasal fossa of the same side.

DR. R. H. Ivy, Philadelphia: There is considerable room for improvement in our present methods of dealing with dental cysts. It is hoped that Dr. MacMillan will continue his researches along the lines of better operative procedures.

Dr. Theodor Bulm, New York: These studies will be quite valuable in our understanding of cysts. Regarding the operative procedure, I disagree with Dr. MacMillan. To

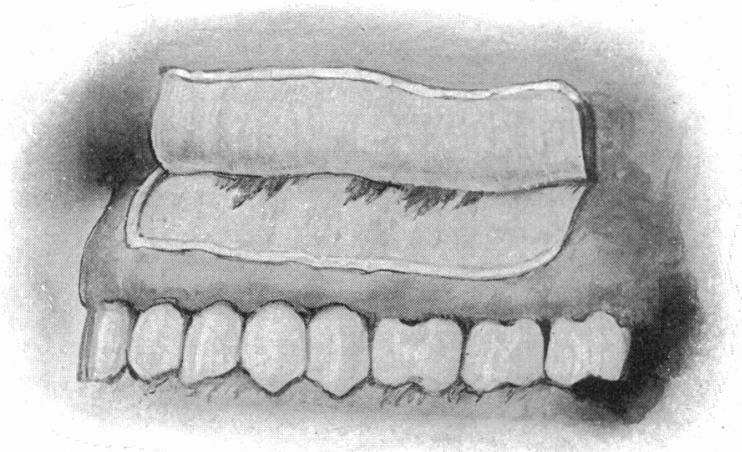
Fig. 5.-The buccal flap should not include the attachments of the
muscles.

open into the antrum and drain into the nasal fossae is an old operation especially performed by the French surgeons, and my objection is that we have no right to open into the healthy maxillary sinus and disturb normal nasal relations to perform an operation which can be accomplished by draining into the mouth. I have in my own practice drained cysts of the maxilla into the nose if the sinus was diseased, because both the sinus and the cyst as well needed attention. The operation which was illustrated by Dr. MacMillan with lantern slides, I personally do not approve of. I find it more advantageous to the dentist who will make a plate later on, if we keep well above the alveolus; even if the teeth have to be removed, they should be retained while operating in cleaning out the cyst, leaving the removal of the teeth as the last part of our operation. In this way we save the alveolus and give the patient as well as the dentist a good foundation for a prosthetic appliance. If the cyst is moderately large the wound can be sutured, and if very large, the flap is replaced in the cyst cavity or sutured to the cyst membrane as indicated.

Dr. Herbert A. Potts, Chicago: When operating in these cases, we should keep in mind the differentiation between cysts and abscesses. The terms as they have been employed in the paper and in the discussion might lead one to think that no clear differentiation was made. Ordinarily the drainage of the cyst is contraindicated; suturing the mucous membrane of the mouth to the membrane of the cyst, I would hardly speak of as a drainage operation. I am in the habit of treating these large cysts as does Dr. Blum. It is a wonderful revelation to see how prone the bones of the face, that is the maxilla and the mandible, are to resume their normal size and contour. After suturing the mucous membrane of the gum to the membrane of the cyst, even though it has fully compressed the antrum, within six or eight months, or a year, the maxilla will be perfectly normal; furthermore, the patient is well in a week, when, if that cyst wall is dissected out, it leaves a suppurating cavity which must be packed for a year or more and then we do not have the restoration as by the other method; furthermore, the replacement is by scar tissue and not by bone. The cyst of the lower jaw acts in the same way, and in the restoration of the alveolar ridge, I have in a few cases simply sutured the cyst wall to the mucous membrane, leaving the denuded teeth in place until a partial restoration had taken place, fearing if I took out those teeth I would take away the bony ridge. After a few weeks there is a new bony formation in

the neighborhood; then teeth are extracted, the result being a firm normal ridge.

Dr. Hugh W. MacMillan, Cincinnati: I tried to emphasize the fact that only extensive cysts involving the maxillary sinus and nasal fossae were being considered. Also, may I again repeat that these extensive radicular cysts do

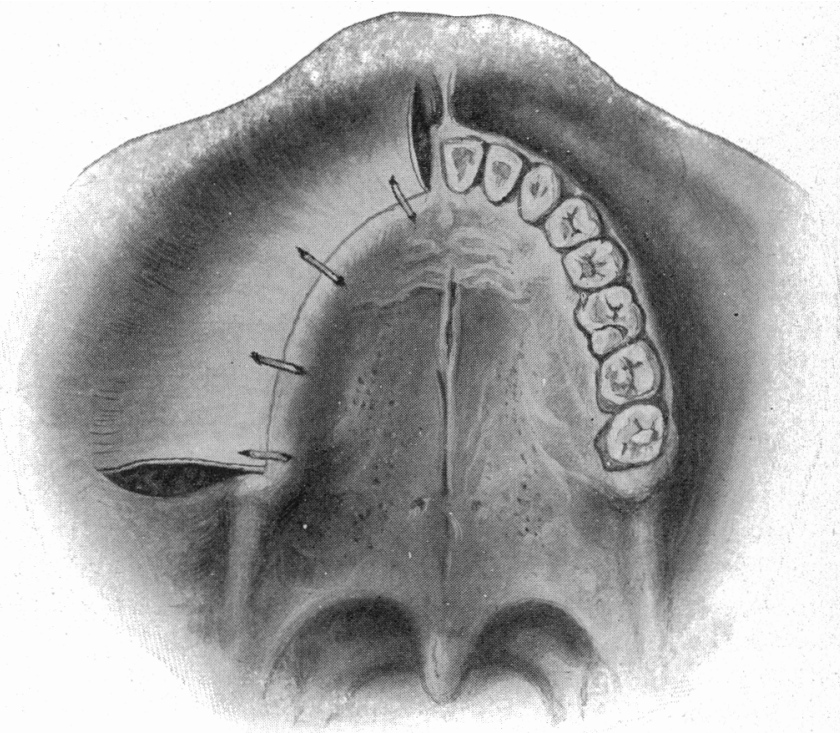

Fig. 7.-The antrum and the cyst cavity are completely separated from the oral cavity by the flap. Drainage is maintained through an opening into the inferior meatus.

occur occasionally around teeth other than the superior lateral incisor, but I believe they occur only when the characteristic anatomic environment of the apex of the tooth is changed to resemble that surrounding the apex of the superior lateral incisor. These anatomic relations equally affect the 
pathology of alveolar abscesses, chronic osteitis or cyst formation. In regard to Dr. Theodor Blum's criticism of the operation, this operation is indicated when the cyst has involved the antrum and nasal cavity, causing great destruction of bone. The end-results show it to be conservative rather than radical. The point on which $I$ expected the greatest discussion has apparently been overlooked. By actual measurement of models it can be demonstrated that, by employing the flap as I have outlined, the eventual depth of the buccal vestibule is the same as before. From the esthetic and prosthetic view point this is the criterion of the success of the operation. The etiology and classification of cyst have not been considered in this paper. An attempt has been made to furnish a further explanation for these extensive cyst formations from an anatomic basis and to present some observations which Dr. Arthur Black in his discussion has said were "points evidently heretofore overlooked."

\section{UROGRAPHY AS A METHOD OF DIAGNOSIS *}

\section{JOHN H. MORRISSEY, M.D. NEW YORK}

The confusing array of terms used to demonstrate the picturing of the various overlapping areas of the genito-urinary tract has been grouped by Dr. Braasch under the term urography.

Perhaps no branch of scientific medicine embraces such diagnostic value and, yet, diversity of methods as urography. In spite of their slow development, modern standardized methods of gastro-intestinal roentgenography now scarcely vary in the slightest detail from a uniform procedure as regards mediums, time element, etc.; on the other hand, urography presents scarcely one uniform detail. Disapproved by many men, actually feared by some, and utilized with extreme caution by others, the method comprises a variety of procedures, including gravity and syringe methods of injection. An infinite number of mediums are used, varying from simple solutions to elaborate paint pot mixtures, with a similarly unstandardized time element as regards injection, distention, roentgenography, and the like.

A discussion of pyelography cannot but encroach on the domain of those who honestly believe that double pyelograms are distinctly contraindicated and my paper does not discuss this issue. We have not had bad results following double pyelograms, and it is our practice to make them. Many of the plates, however, which are inclucled in this demonstration are single pyelograms. Nor are we insistent on the use of sodium iodid as a medium. Our experience, however, has borne out our preference for the solution.

Careful studies of sodium iodid convinced Cameron in 1914 that it was ideal for pelvic injection, and he demonstrated its relative nontoxicity. Later studies by Lowsley and Muller, recently reported, bore out his observation, to the exclusion of numerous other mediums tried. Injected bladder and kidneys showed only a cloudy urine as the result of injection. This was likewise produced by kidney trauma, and hence could not be adversely interpreted. No relative discrepancy was noted in the effect of the gravity or syringe method of injection, except in the accuracy of the former. Consequently we have adopted, as a medium, 20 per

* From the Urological Department (James B. Brady Foundation) of the New York Hospital.

A Read before the Section on Urology at the Seventy-Third Annua cent. solution of sodium iodid, introduced by springe, as our standard procedure.

Thirty-nine urologic services and departments were questioned as to the routine method of urography. Sixteen were using sodium thorium nitrate as a medium, ten were using sodium bromid, eight were using sodium iodid, and the remainder used various other solutions.

Indispensable, it would seem, for the proper performance of pyelography is the proximity of the roentgen-ray outfit of the cystoscopic table. A review of the methods available for roentgen ray revealed that only eleven, or 14 per cent., had facilities for roentgenographing the patient immediately at the end of the ureteral catheterization. In nineteen hospitals, the table was removed or the patient shifted to a hospital truck and transported a varying distance to the roentgen-ray department, the cystoscope having been taken out and catheters left in situ. I believe that loss of time in this procedure is responsible for many of the difficulties of pyelography. Delays of one sort or another in the roentgen-ray room naturally entail further ureteral trauma from the continued presence of the catheter, with only too frequent reflex inhibition of the kidney, which is subsequently less resistant to the introduction of the urographic medium. In this connection a standard of procedure would certainly insure more uniform results. I do not feel that it is fair to credit urography with harmful results obtained under these conditions.

The arrangement illustrated in Figure 1 is, to my mind, ideal for a cystoscopic and roentgenographic outfit. This is in use at the James B. Brady Foundation of the New York Hospital and was installed at a minimum of expense. For a smaller outfit, the entire equipment would obviously cost much less.

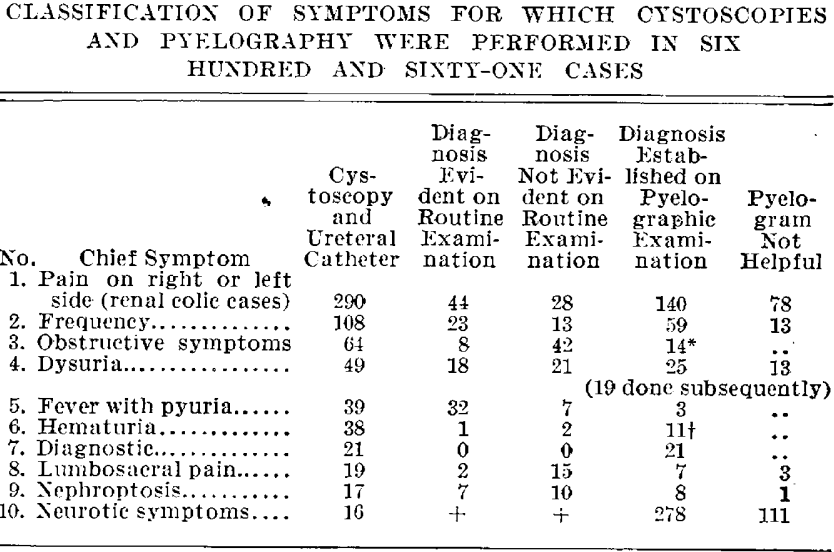

* Includes numerous cases of obstruction at vesical neek.

+ Inclurles various cases of bladder tumor.

In this connection, it might be well to state that our three most serious reactions following urologic procedures occurred in instances in which pyelography had not been used and only ureteral catheterization had been performed. Two of these patients developed a complete suppression and subsequently recovered. Numerous observations are at hand that simple observation cystoscopy has been fraught with serious consequences. In other cases, if pyelography has been done, the ill effects are attributed to the pyelography, whereas the catheterization of ureters is equally, if not more greatly, at fault. Yet one would not think of interdicting ureteral catheterization on this account. 